\title{
IDENTIDAD CULTURAL, ACTITUDES POLÍTICAS Y VALORES SOCIOCULTURALES EN TIJUANA, CIUDAD JUÁREZ, NUEVO LAREDO Y SAN LUIS POTOSÍ
}

\author{
Por \\ José Carlos Lozano Rendón*
}

\begin{abstract}
RESUMEN
Mediante el uso de la base de datos que constituye la Encuesta Socioeconómica Anual de la Frontera 1987 (ESAF 87) de El Colegio de la Frontera Norte, el presente artículo discute las actividades políticas hacia Estados Unidos y ciertos valores familiares tradicionales de México en los encuestados de Tijuana, Ciudad Juárez, Nuevo Laredo y San Luis Potosí.

Mediante el análisis de las escalas de actitudes sobre el sistema capitalista, sobre los Estados Unidos y sobre las premisas socioculturales definidas por Rogelio Díaz Guerrero, como características de elementos de la cultura tradicional mexicana, se concluye que la clase social y el sexo constituyen variables más útiles para predecir la pérdida de identidad cultural que la proximidad geográfica con los Estados Unidos.
\end{abstract}

\begin{abstract}
Through the utilization of the data base constituted by the 1987 Annual Socio-Economic Border Survey, made by El Colegio de la Frontera Norte, this article discusses the political activities towards USA and certain Mexican traditional familiar values found in the people surveyed in Tijuana, Ciudad Juárez, Nuevo Laredo and San Luis Potosí.

Using the analysis of the scale of attitudes on the cupitalist system, on the United States and on the socio-economical premises defined by Rogelio Diaz Guerrero as characteristic of elements of the Mexican traditional culture, it is concluded that in order to predict the loss of cultural identity, social class and sex are more useful variables than the geographical proximity to the United States.
\end{abstract}

La inminencia de un acuerdo de libre comercio entre México y los Estados Unidos ha hecho surgir, en algunos sectores, la preocupación por una posible pérdida de identidad cultural en la población mexicana. Esta preocupación, externada desde hace mucho tiempo en referencia a la

\footnotetext{
* Investigador titular en El Colegio de la Frontera Norte en Nuevo Laredo, Tamaulipas.
} 
frontera norte del país, se basa en la creencia de que a mayor contacto y vinculación con los Estados Unidos, mayor deterioro en los valores y actitudes que conforman el sentido de pertenencia y lealtad a la cultura mexicana. Dadas las condiciones asimétricas en la relación bilateral con los Estados Unidos y al histórico predominio mundial de los productos comunicacionales de este último (películas, series de televisión, música, noticias, moda, etcétera), se considera que la mayor apertura de México hacia ese país redundará en la erosión paulatina e inexorable de su patrimonio cultural.

Los temores hacia la penetración ideológica norteamericana no son exclusivos de México, Canadá, a pesar de ser un país industrializado con mayor equilibrio en su relación bilateral con Estados Unidos, ha experimentado una situación similar. En las discusiones para establecer el tratado de libre comercio con su vecino, Canadá incluyó de manera sobresaliente el tema de la preservación de su identidad nacional. Desaulniers (1987) menciona que los funcionarios canadienses hicieron referencias constantes a la salvaguarda de las manifestaciones culturales autóctonas de su país, al grado de exasperar a los negociadores norteamericanos. En esa misma nación, el debate original de los francófonos de Quebec por preservar su idioma y su cultura se ha extendido en la última década al impacto negativo de las películas, programas televisivos, revistas, periódicos y noticias provenientes de los Estados Unidos.

En este contexto, no es extraño que funcionarios, académicos y grupos de diversas tendencias expresen en México sus temores a una desnacionalización producto de la "avasalladora" penetración cultural norteamericana. Desde antes de las discusiones sobre el acuerdo de libre comercio, como lo señala Jorge Castañeda (1989: 413), tanto los nacionalistas de izquierda como los de derecha han condenado con similar ímpetu la creciente "norteamericanización" del país, fenómeno que — según ellosnos lleva "al desastre por la vía de la pérdida de la identidad, de la pureza y de los valores tradicionales de nuestra nación". En la circunstancia de una mayor apertura de las fronteras nacionales a flujos de bienes y servicios, capital y trabajo, así como de información y cultura originados en Estados Unidos, las conjeturas sobre el impacto en la identidad cultural han adquirido en ocasiones tintes apocalípticos.

La experiencia de la frontera norte en el ámbito de la identidad nacional, sin embargo, ofrece algunas evidencias que podrían tranquilizar a quienes externan dichos temores. Los hallazgos obtenidos mediante encuestas y otras técnicas de investigación por estudiosos de esta región del país, en los últimos años, muestran consistentemente que su intensa vinculación económica y geográfica con los Estados Unidos no ha redun- 
dado en una pérdida de identidad cultural o en diferencias significativas en percepciones y actitudes sobre México y los Estados Unidos.

Una de las primeras investigaciones empíricas sobre identidad nacional en la frontera de México con los Estados Unidos fue realizada en 1982 por un equipo de investigadores (Cfr. Bustamante, 1983) del entonces Centro de Estudios de la Frontera Norte de México (ahora El Colegio de la Frontera Norte). El estudio se basó en una encuesta a 1,330 jóvenes de tres ciudades fronterizas (Tijuana, Ciudad Juárez y Matamoros) y de la Ciudad de México, e incluyó una prueba sociopsicológica diseñada por el doctor Rogelio Díaz Guerrero para medir el "carácter nacional" de los fronterizos. A partir de la lejanía o cercanía de las respuestas de los entrevistados respecto de premisas socioculturales derivadas de dichos, consejas y valores de la cultura tradicional mexicana, Díaz Guerrero estableció un índice de mayor o menor nivel de identidad nacional de los informantes. Los resultados indicaron que al margen de la cercanía geográfica con los Estados Unidos, los jóvenes de clase alta en todas las ciudades incluidas en la muestra (identificados como del sector I en el estudio) tendían a identificarse menos con las premisas socioculturales relacionadas con el "carácter nacional" que los de clase media y baja (sector II). Según Bustamante, con base en los hallazgos podía concluirse que "hay una pérdida de 'identidad nacional' entre los jóvenes del sector I de las cuatro poblaciones" (1983:46). Comparando a los jóvenes de las tres ciudades fronterizas con los de la Ciudad de México, sin embargo, Bustamante advirtió una diferencia significativa entre ambos grupos:

Al comparar los datos correspondientes a las tres ciudades fronterizas con los del Distrito Federal encontramos que hay una mayor retención de los valores de las tradiciones culturales mexicanas entre los jóvenes de las ciudades fronterizas que entre los del Distrito Federal (...) Este hallazgo viene a confirmar lo que hemos encontrado en otra investigación anterior del CEFNOMEX en el sentido de que hay una mayor retención de los valores de las tradiciones culturales mexicanas en la frontera norte que en la Ciudad de México. Este hallazgo derivado de una investigación científica es claramente contrario a una creencia generalizada de la existencia de un proceso de desnacionalización de la población fronteriza del norte del país. (Bustamante, 1983: 46-47).

El presente trabajo constituye una réplica del estudio realizado en 1983 por los investigadores del entonces CEFNOMEX en su parte correspondiente a la escala del carácter nacional diseñada por Díaz Guerrero. Tomando como base los resultados de la escala corta de premisas socioculturales 
incluida en la Encuesta Socioeconómica Anual de la Frontera (ESAF) 1987 , el análisis pretende determinar si los hallazgos de 1983 seguían siendo válidos en 1987 y si lo concluido sobre Matamoros y la Ciudad de México podría extrapolarse a Nuevo Laredo y San Luis Potosí.

Por otro lado, el presente estudio añade el análisis de una escala relativa a las percepciones generales del capitalismo y de Estados Unidos en los habitantes de la frontera norte, no incluida en la encuesta de 1983. La posibilidad de conocer si los habitantes fronterizos poseen percepciones más favorables hacia el capitalismo y/o hacia Estados Unidos que los residentes en San Luis Potosí permite contar con un nuevo recurso con qué cotejar los resultados de la escala del carácter nacional.

En la investigación de 1983 se le da particular importancia a la variable de clase social, ya que la pertenencia a un determinado sector - con los beneficios materiales e intelectuales que conlleva o excluye- parece resultar determinante en el grado de identificación del individuo con la cultura tradicional mexicana o con la ideología norteamericana. La situación hegemónica de las clases altas, como argumentan numerosos científicos sociales, propicia su identificación con los valores característicos del sistema capitalista, mientras que la situación de opresión y subordinación de las clases bajas fomenta la supervivencia de elementos tradicionales de la cultura popular (Cfr. Stavenhagen, 1984 y Bonfil Batalla, 1982). Aunque la relación de las clases privilegiadas con la ideología norteamericana o capitalista está lejos de ser mecánica e inexorable, parece obvio que su situación en la estructura social las orilla naturalmente a identificarse con los supuestos básicos que la legitiman. Por ello, como señala Durán, las clases dominantes reproducen su cultura de clases propia y los "patrones, complejos y modalidades de culturas ajenas, externas, provenientes de los centros de dominación mundial" (1984:75). Las clases populares, por su parte, tienden a preservar rasgos culturales y prácticas sociales que discrepan directa o indirectamente de las hegemónicas. Así, rechazan determinados valores y actitudes de los sectores dominantes y se adhieren a elementos subalternos, a tradiciones y visiones alternativas del mundo. Este proceso, sin embargo, es sumamente complejo, amén de contradictorio. Como señala García Canclini, "no existen sectores que se dediquen full-time a construir la hegemonía, otros entregados al consumismo y otros tan concientizados que viven sólo para la resistencia y el desarrollo autónomo de una existencia popular alternativa" (1987: 30). De ahí que, además de la clase social como variable independiente, se hace necesario incluir otras como el sexo, la edad y el grado de escolaridad, así como de la proximidad geográfica con los Estados Unidos en el caso de la región fronteriza. 
El sexo, en particular, parece constituir una variable importante en el mantenimiento o rechazo de los elementos característicos de la cultura nacional. Las diferencias significativas en la adjudicación de roles y conductas a cada sexo en la cultura mexicana parecería propiciar que las mujeres se identifiquen más con la cultura nacional que los hombres de su mismo estrato socioeconómico.

Por último, la variable de proximidad geográfica con los Estados Unidos es esencial para determinar si, como afirman algunos, los fronterizos presentan mayores síntomas de penetración cultural extranjera que los mexicanos del interior del país. En este sentido, la inclusión de la Ciudad de México en la encuesta de 1983 y de San Luis Potosí en la ESAF 1987 permiten determinar si esta variable es significativa o no en la conformación del carácter y la identidad cultural en la frontera norte. De acuerdo a los resultados obtenidos en estudios anteriores realizados por El Colegio de la Frontera Norte (COLEF), esta cercanía geográfica con Estados Unidos no parece ejercer una influencia determinante, ya que la clase social, en cualquier parte del país, tiende a motivar a los miembros de la clase alta a identificarse con la idcología y los valores estadounidenses.

Con base en lo anterior, el presente análisis plantea las siguientes hipótesis: 1) la distancia geográfica con los Estados Unidos no se asocia con una mayor o menor afinidad con los supuestos básicos del modo de producción capitalista; 2) la distancia geográfica con los Estados Unidos no se asocia con una percepción más o menos favorable de Estados Unidos; 3) la distancia geográfica con los Estados Unidos no se asocia con una mayor o menor afinidad con los valores tradicionales de la cultura mexicana; 4) a mayor clase social, mayor afinidad con los supuestos básicos del modo de producción capitalista; 5) a mayor clase social, más favorable la percepción de los Estados Unidos; 6) a mayor clase social, mayor la lejanía hacia los valores tradicionales de la cultura mexicana; 6) las personas de sexo masculino tienden más que las de sexo femenino a tener actitudes políticas favorables hacia el modo de producción capitalista; 7) las personas de sexo masculino tienden más que las de sexo femenino a tener actitudes políticas favorables hacia los Estados Unidos; 8) las personas de sexo femenino tienden a identificarse más que las de sexo masculino con las premisas socioculturales de la cultura tradicional mexicana.

Con la escala de premisas socioculturales de Díaz Guerrero y la de actitudes políticas, la ESAF 1987 no pretendió definir de una manera completa y consistente lo que constituye la "identidad cultural" de los mexicanos. La complejidad y el dinamismo del concepto hace punto menos que imposible la posibilidad de definirlo concretamente o de operacionalizarlo. Más bien, ambas escalas constituyen una aproximación 
limitada y parcial a un fenómeno extraordinariamente difícil y elusivo. Consecuentemente, el presente trabajo tampoco pretende obtener respuestas contundentes y finales a las hipótesis planteadas arriba. Los hallazgos deberán tomarse como indicadores limitados, pero confiables, de algunos rasgos que sí contribuyen, aunque sea de manera incompleta, a la definición y explicación del concepto "identidad cultural". Como señala Bustamante en su reporte de la encuesta de 1983 sobre la escala del carácter nacional, ésta "no mide todo aquello que podría o debería incluir una noción completa de identidad nacional... [pero] se trata de una aproximación que, sin embargo, contiene elementos sin los cuales no se podría hablar de una identidad nacional en aquello que se refiere a valores y tradiciones característicos de la cultura mexicana" (1983: 44).

\section{METODOLOGÍA}

El análisis realizado en este estudio se centra en los datos recabados por El Colegio de la Frontera Norte a través de la Encuesta Socioeconómica Anual de la Frontera (ESAF) realizada en el mes de septiembre de 1987 en las ciudades de Tijuana, Baja California, Ciudad Juárez, Chihuahua, Nuevo Laredo, Tamaulipas y San Luis Potosí. Esta encuesta incluyó un cuestionario de hogares con variables demográficas, laborales, migratorias y educativas, y otro individual que se centró en dos escalas: la de premisas socioculturales relativas al carácter y la identidad nacional desarrollada por Díaz Guerrero y aplicada en anteriores encuestas del COLEF, y la de actitudes políticas, desarrollada por el doctor Tonatiúh Guillén López, director del Departamento de Estudios Urbanos del COLEF e incluida por primera vez en ese año de 1987. En la distribución y levantamiento de la muestra de la ESAF 1987 se siguieron los procedimientos metodológicos para encuestas demográficas del Instituto Nacional de Estadística, Geografía e Informática (INEGI) (González, 1990).

El cuestionario individual le fue aplicado a un total de 5,809 personas distribuidas de la siguiente manera: Nuevo Laredo, 1,385 casos; Ciudad Juárez, 1,618; Tijuana, 1,225; y San Luis Potosí, 1,581. Las variables incluidas en el cuestionario y tomadas en cuenta para el presente estudio fueron: municipio, sector socioeconómico, sexo, edad y los diferentes reactivos que compusieron las escalas de carácter nacional y de actitudes políticas.

La escala de premisas socioculturales comprendió cuatro factores cuyos respectivos items, en anteriores encuestas, demostraron tener un peso factorial superior a .40:1) creencia en que uno debe tener temor a la autoridad de los padres; 2 ) creencia en que el respeto hacia los padres es 
más importante que el amor; 3) creencia en la importancia de obediencia a los padres; y 4) creencia en la superioridad masculina (machismo). Estas premisas, determinadas por Díaz Guerrero como características de la mayoría de los mexicanos en todo el país, se tomaron como base para determinar si los habitantes de la frontera norte presentaban un alejamiento significativo hacia ellas. Aunque es muy cuestionable que los cuatro factores constituyen una operacionalización adecuada y comprensiva de la "identidad nacional" de los mexicanos, sí ofrecen un punto de partida útil para encontrar semejanzas, o diferencias, entre las creencias más tradicionales en el ámbito de las relaciones familiares de los habitantes del interior y los del norte del país. Los reactivos incluyeron afirmaciones tales como "es más importante obedecer al padre que amarlo", "muchos hijos temen a sus padres", "algunas veces un hijo no debe obedecer a su madre", contestándose en opciones estilo Lickert desde "muy de acuerdo" hasta "muy en desacuerdo".

La escala de actitudes políticas, por otra parte, fue diseñada para detectar las tendencias dominantes ( y su perfil) en la estructuración de los valores políticos de la población fronteriza en un momento determinado. Mediante diversas proposiciones relativas a las relaciones más generales del modo de producción capitalista y a situaciones concretas de la formación social y del poder político, la escala permitió conocer la percepción de los fronterizos sobre el sistema capitalista en abstracto y sobre los Estados Unidos en particular. Las actitudes políticas sobre los dos puntos anteriores se reflejaron en las respuestas a 13 reactivos planteados en forma de afirmaciones con opciones que iban desde "acuerdo" hasta "desacuerdo". Nueve de esas 13 proposiciones formaron, para los propósitos del presente estudio, la variable "actitud política hacia el modo de producción capitalista":

1) No existe libertad en los países capitalistas.

2) Los mejores gobernantes serían los empresarios.

3) La riqueza se obtiene gracias al esfuerzo personal.

4) Los mejores gobernantes serían los campesinos y obreros.

5) Las personas son libres en los países comunistas.

6) Es mejor que la economía sea controlada por el gobierno.

7) El socialismo es la mejor opción para México.

8) Los empresarios son los principales causantes de la crisis.

9) No hay democracia en los países capitalistas.

Las cuatro afirmaciones restantes - "El país más democrático del mundo es Estados Unidos", "Estados Unidos ha obstaculizado el desarro- 
llo de México", "Las elecciones en México deberían ser como en Estados Unidos", "Los problemas en Centroamérica los causan grupos comunistas"- formaron por su parte la variable "actitud política hacia Estados Unidos". De la misma manera en que la escala de premisas socioculturales no agota ni es suficiente para definir la identidad nacional, tampoco estas proporciones son suficientemente incluyentes en lo que respecta a la influencia de la ideología política norteamericana en los habitantes de la región. Sin embargo, ofrecen una aproximación que consideramos válida al análisis de ese impacto ideológico que se presupone existe, pero que pocas veces se ha visto sujeto a comprobación. Para el análisis de los datos se procedió de la siguiente manera. En primer lugar, se agruparon las respuestas de los diferentes reactivos que componían cada uno de los factores o variables generales emanadas de las dos escalas (cuatro en el caso de la de premisas socioculturales, dos en el caso de la de actitudes políticas) para cada ciudad incluida en la ESAF 1987. Posteriormente, se realizaron análisis de varianza simples para determinar diferencias estadísticas en las medias obtenidas para cada población y factor por estrato socioeconómico, y pruebas $t$ para las medias obtenidas en cada ciudad y factor por sexo y edad. Mediante esas mismas pruebas, se contrastó el grado de significación estadística relativa a las diferencias encontradas entre las cuatro ciudades entre los mismos grupos de sexo, edad y sector socioeconómico.

\section{RESULTADOS}

Con respecto a la primera hipótesis que pronosticaba que la distancia geográfica con los Estados Unidos no se asocia con una mayor o menor afinidad con los supuestos básicos del modo de producción capitalista, el cuadro 1 permite establecer su comprobación. Aunque sí se daban diferencias estadísticamente significativas entre cada una de las poblaciones, la proximidad geográfica no parecía ser la explicación, ya que los habitantes de Tijuana y Nuevo Laredo tenían una percepción menos favorable del modo de producción capitalista que los de San Luis Potosí. Únicamente los encuestados de Ciudad Juárez percibían a dicho sistema económico más favorable, con un promedio en la escala de respuesta de 1.94 ("de acuerdo" con las afirmaciones favorables hacia los Estados Unidos, aunque cercano al "tal vez").

Una situación similar se presentaba para la hipótesis relativa a percepciones favorables o desfavorables sobre Estados Unidos en la frontera norte debido a la cercanía con ese país. El cuadro 2 muestra que la población de San Luis Potosí presentaba actitudes políticas más favorables 
CUADRO 1. Análisis de varianza simple de percepciones favorables al sistema capitalista en habitantes de Nuevo Laredo, Ciudad Juárez, Tijuana y San Luis Potosí: 1987.

\begin{tabular}{lrcccc}
\hline Ciudad & Media & $\begin{array}{c}\text { Desviación } \\
\text { estándar }\end{array}$ & n & F & $\begin{array}{c}\text { Signific. } \\
\text { de la F }\end{array}$ \\
\hline Cd. Juárez & $\begin{array}{r}17.50 \\
1.94^{\circ}\end{array}$ & 4.5 & 1598 & 68.9 & $.0000^{\mathrm{b}}$ \\
San Luis Potosí & $\begin{array}{r}18.00 \\
2.00\end{array}$ & 4.7 & 1536 & & \\
Tijuana & $\begin{array}{r}2.60 \\
2.07\end{array}$ & 5.0 & 1216 & & \\
Nuevo Laredo & 20.00 & 5.6 & 1372 & & \\
& 2.22 & & & & \\
\end{tabular}

FUENTE: Encuesta Socioeconómica Anual de la Frontera 1987 (ESAF) de El Colegio de la Frontera Norte.

"Media de las 9 proposiciones que componen la variable "actitudes favorables hacia el modo de producción capitalista" (valor mínimo, 9; máximo, 27). A menor cifra, mayor identificación con las proposiciones favorables al capitalismo.

${ }^{b}$ Cada población es significativamente diferente a las otras tres.

'Promedio en la escala de respuesta: 1 "De acuerdo", 2 "Tal vez", 3 "En desacuerdo".

hacia Estados Unidos que las de Tijuana y Nuevo Laredo, mientras que la de Ciudad Juárez seguía siendo la única que la superaba en favoritismo. ${ }^{1}$

Con respecto a la afirmación de que la distancia geográfica con los Estados Unidos no se asocia con una mayor o menor afinidad con los valores tradicionales de la cultura mexicana, el cuadro 3 muestra las medias obtenidas en la escala de premisas socioculturales para las poblaciones en general. ${ }^{2}$ Como se puede observar, por lo menos una ciudad

1 A pesar de ello, la media de 8.4 dividida entre las cuatro proposiciones favorables a Estados Unidos señalaba que los informantes de Ciudad Juárez se ubicaban en el 2.1 del continuo 1 (acuerdo) a 3 (desacuerdo). Es decir, tendían a contestar "tal vez" en lugar de estar de acuerdo con las afirmaciones positivas sobre Estados Unidos.

2 Para conformar la escala, los datos se agruparon inicialmente por cada uno de los factores: temor a la autoridad de los padres (4 reactivos), respeto a los padres sobre el amor (3 reactivos), obediencia afiliativa sobre autoafimación activa (7 reactivos) y superioridad masculina o machismo (4 reactivos). Posteriormente, estos resultados se agruparon a su vez para reportarse como hallazgos de la escala de premisas socioculturales. Así, las medias reportadas para esta última constituyen el promedio de respuestas individuales a las 18 afirmaciones incluidas en la escala tipo Lickert que se utiliz6 en la RSAP 87. 
CUADRO 2. Análisis de varianza simple de percepciones favorables hacia Estados Unidos en habitantes de Nuevo Laredo, Ciudad Juárez, Tijuana y San Luis Potosí: 1987.

\begin{tabular}{lccccc}
\hline Ciudad & Media $^{\mathrm{a}}$ & $\begin{array}{c}\text { Desviación } \\
\text { estándar }\end{array}$ & $\mathrm{n}$ & $\mathrm{F}$ & $\begin{array}{c}\text { Signific. } \\
\text { de la F }\end{array}$ \\
\hline Cd. Juárez & $\begin{array}{c}8.4 \\
(2.10)^{\mathrm{c}}\end{array}$ & 2.3 & 1598 & 68.9 & $.0000^{\mathrm{b}}$ \\
San Luis Potosí & $\begin{array}{c}8.7 \\
(2.17)\end{array}$ & 2.7 & 1536 & & \\
Tijuana & $\begin{array}{c}9.0 \\
(2.25)\end{array}$ & 2.5 & 1216 & & \\
Nuevo Laredo & $\begin{array}{c}9.3 \\
(2.32)\end{array}$ & 3.0 & 1372 & & \\
& $(2.3)$ & & & & \\
\hline
\end{tabular}

FUENTE: Encuesta Socioeconómica Anual de la Frontera 1987 (ESAF) de El Colegio de la Frontera Norte.

a Media de las 4 proposiciones que componen la variable "actitudes favorables hacia Estados Unidos" (valor mínimo, 4; máximo, 20). A menor cifra, mayor identificación con las proposiciones favorables a los Estados Unidos.

${ }^{\mathrm{b}}$ Cada población es significativamente diferente a las otras tres.

"Promedio en la escala de respuesta: 1 "De acuerdo", 2 "Tal vez", 3 "En desacuerdo".

fronteriza, Nuevo Laredo, mantenía la misma cercanía hacia las premisas consideradas por Díaz Guerrero como indicador del carácter nacional. Tijuana permanecía en un segundo lugar y Ciudad Juárez mostraba la mayor lejanía de las cuatro ciudades hacia dichas premisas. A pesar de diferencias estadísticamente significativas entre estas dos últimas poblaciones y las de Nuevo Laredo y San Luis Potosí, las medias de las cuatro promediaban en la opción "de acuerdo" de la escala de Lickert. ${ }^{3}$ Es decir, la mayoría de los encuestados de las cuatro ciudades estaban de acuerdo con las afirmaciones relacionadas con las actitudes familiares y psicológicas tradicionales en la cultura mexicana.

3 La escala incluía como opciones de respuesta las siguientes categorías: " 1 . muy de acuerdo", “2. de acuerdo", "3. tal vez", “4. en desacuerdo", "5. muy en desacuerdo". Si las medias reportadas en los cuadros las dividimos entre 18 -número de reactivos que en conjunto componen la escala de premisas socioculturales - obtenemos la ubicación de la media en el continuo de opciones de respuesta. Por ejemplo, la media de 49.4 de San Luis Potosí, dividida entre 18 da 2.74, es decir, "de acuerdo". La media de 53.9 de Ciudad Juárez, entre 18, da 2.99, es decir "de acuerdo" casi llegando al "tal vez". 
CUADRO 3. Análisis de varianza simple de la escala de premisas socioculturales en habitantes de Nuevo Laredo, Ciudad Juárez, Tijuana y San Luis Potosí: 1987.

\begin{tabular}{lccccc}
\hline Ciudad & Media $^{\mathrm{a}}$ & $\begin{array}{c}\text { Desviación } \\
\text { estándar }\end{array}$ & $\mathrm{n}$ & $\mathrm{F}$ & $\begin{array}{c}\text { Signific. } \\
\text { de la F }\end{array}$ \\
\hline San Luis P. & $\begin{array}{c}49.45 \\
(2.75)^{\mathrm{c}}\end{array}$ & 8.5 & 1581 & 74.0 & $.0000^{\mathrm{b}}$ \\
Nuevo Laredo & $\begin{array}{c}50.0 \\
(2.78)\end{array}$ & 8.0 & 1385 & & \\
Tijuana & $\begin{array}{c}51.9 \\
(2.88)\end{array}$ & 10.3 & 1225 & & \\
Ciudad Juárez & $\begin{array}{c}53.9 \\
(2.99)\end{array}$ & 9.9 & 1618 & & \\
& & & & & \\
\hline
\end{tabular}

FUENTE: Encuesta Socioeconómica Anual de la Frontera 1987 (ESAF) de El Colegio de la Frontera Norte.

a Media de las 18 proposiciones que componen la escala "premisas socioculturales sobre el carácter nacional" (valor mínimo, 18; máximo, 90). A menor cifra, mayor identificación con las premisas tradicionales de la cultura mexicana definidas por Díaz Guerrero.

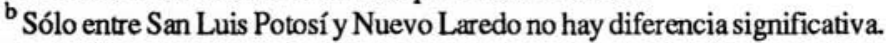

"Promedio en la escala de respuesta: 1 "de acuerdo", 2 "tal vez", 3 "en desacuerdo".

Con respecto a las hipótesis relativas a la importancia de la clase social como variable independiente en las actitudes favorables o desfavorables hacia el sistema capitalista, hacia Estados Unidos y hacia las premisas socioculturales de la familia mexicana, $\operatorname{los}$ cuadros 4 a 6 dan una idea clara de su pertinencia.

Con respecto a la hipótesis que plantea que a mayor clase social mayor afinidad con los supuestos básicos del modo de producción capitalista, el cuadro 4 muestra la comparación de las medias obtenidas entre los sectores de cada población en particular. En las cuatro ciudades, sin importar la proximidad geográfica a Estados Unidos, se advierte que los sectores I mantenían actitudes significativamente más favorables hacia el sistema capitalista que los sectores III. Los sectores II, por su parte, presentaban un comportamiento diverso. En San Luis Potosí y Ciudad Juárez sí se encontraban en la posición intermedia entre sus sectores I y II, pero en Tijuana no se diferenciaban del sector III y en Nuevo Laredo se identificaban en grado significativamente mayor con el sistema capitalista que su mismo 
CUADRO 4. Análisis de varianza simple de actitudes favorables al sistema capitalista entre los tres sectores socioeconómicos de Nuevo Laredo, Ciudad Juárez, Tijuana y San Luis Potosí: 1987.

\begin{tabular}{|c|c|c|c|c|}
\hline Estrato & $\begin{array}{l}\text { Nuevo } \\
\text { Laredo }\end{array}$ & $\begin{array}{l}\text { Ciudad } \\
\text { Juárez }\end{array}$ & Tijuana & $\begin{array}{c}\text { San Luis } \\
\text { Potosí }\end{array}$ \\
\hline Sector I & $\begin{array}{c}19.4^{\mathrm{a}} \\
2.1^{\mathrm{b}} \\
(6.1)^{\mathrm{c}} \\
\mathrm{n}=348\end{array}$ & $\begin{array}{c}15.9 \\
1.8 \\
(4.8) \\
\mathrm{n}=335\end{array}$ & $\begin{array}{c}16.8 \\
1.9 \\
(5.2) \\
\mathrm{n}=193\end{array}$ & $\begin{array}{c}15.0 \\
1.7 \\
(3.9) \\
\mathrm{n}=320\end{array}$ \\
\hline Sector II & $\begin{array}{c}18.5 \\
2.0 \\
(4.8) \\
\mathrm{n}=604\end{array}$ & $\begin{array}{c}17.1 \\
1.9 \\
(4.4) \\
n=714\end{array}$ & $\begin{array}{c}18.9 \\
2.1 \\
(4.8) \\
n=533\end{array}$ & $\begin{array}{c}18.3 \\
2.0 \\
(4.3) \\
n=571\end{array}$ \\
\hline Sector III & $\begin{array}{c}22.3 \\
2.5 \\
(5.5) \\
m=433\end{array}$ & $\begin{array}{c}18.9 \\
2.1 \\
(4.0) \\
\mathrm{n}=569\end{array}$ & $\begin{array}{c}18.9 \\
2.1 \\
(4.9) \\
\mathrm{n}=499\end{array}$ & $\begin{array}{c}19.2 \\
2.1 \\
(4.9) \\
\mathrm{n}=690\end{array}$ \\
\hline Valor de F & 62.8 & 55.7 & 14.1 & 97.3 \\
\hline $\begin{array}{l}\text { Significanc } \\
\text { de la F }\end{array}$ & $.0000^{\mathrm{d}}$ & $.0000^{\mathrm{e}}$ & $.0000^{f}$ & $.0000^{\mathrm{g}}$ \\
\hline FUENTE: En & $\begin{array}{l}\text { a Socioeco } \\
\text { de la Fron } \\
\text { a de las } 9 \\
\text { talistas" (v } \\
\text { cación con } \\
\text { edio en la } \\
\text { esacuerdo" } \\
\text { ación están } \\
\text { sector es si } \\
\text { Tukeyb) } \\
\text { sector es sig } \\
\text { los sectores } \\
\text { sector es sig }\end{array}$ & $\begin{array}{l}\text { a Anual } \\
\text { orte. } \\
\text { iciones c } \\
\text { ínimo, 9; } \\
\text { posicion } \\
\text { de resp } \\
\text { ativamen } \\
\text { ivamente } \\
\text { no hay d }\end{array}$ & $\begin{array}{l}\text { Fronterc } \\
\text { mponen } 1 \\
\text { no, 21). } \\
\text { rables al } \\
1 \text { "de ac } \\
\text { rente de lo } \\
\text { inte de los } \\
\text { cia signifi }\end{array}$ & $\begin{array}{l}7 \text { (ESAF) de El } \\
\text { able "actitudes } \\
\text { or cifra, mayor } \\
\text { lismo. } \\
\text { ". } 2 \text { "tal vez". } \\
\text { s dos (procedi- } \\
\text { dos (P. Tukeyb) } \\
\text { (P. Tukeyb) } \\
\text { dos (P. Tukeyb) }\end{array}$ \\
\hline
\end{tabular}


sector I. Mientras que das clases altas ubicaban su respuesta en el límite superior de la opción "de acuerdo", las clases bajas lo hacían en el límite inferior de la opción "tal vez", demostrando que no mantenían actitudes tan favorables hacia el sistema capitalista como los del sector I. De esta manera, la hipótesis de que a mayor clase social mayor identificación con dicho modo de producción parecía quedar corroborada, aunque los sectores II mostraban tendencias desiguales al respecto.

En cuanto a las actitudes hacia Estados Unidos en particular, los sectores socioeconómicos de las cuatro ciudades tendían a mostrar un comportamiento distinto. El cuadro 5 muestra que el promedio de todos los sectores en las cuatro ciudades se ubican en el límite inferior de la opción "tal vez", lo que mostraba en los sectores I actitudes menos favorables hacia Estados Unidos que las que manifestaban hacia el sistema capitalista. Con la excepción de Ciudad Juárez, todos los sectores I presentaban mayor proximidad con las afirmaciones favorables hacia Estados Unidos que los sectores III. De esta manera, la hipótesis de que a mayor clase social mayor afinidad con los Estados Unidos también resultaba válida, aunque los sectores II de nuevo manifestaban un comportamiento irregular en las ciudades fronterizas, identificándose más que sus sectores I con las proporciones favorables a los Estados Unidos (caso de Nuevo Laredo y Tijuana), o menos que su sector III (caso de Ciudad Juárez). Los sectores socioeconómicos II de San Luis Potosí, al igual que en las actitudes hacia el capitalismo, eran los únicos que adoptaban la posición intermedia pronosticada por la hipótesis.

El cuadro 6 muestra una situación muy similar a la anterior en relación a las premisas socioculturales. De nuevo, los sectores I de cada población diferían significativamente de los sectores III, con estos últimos manifestando mayor cercanía con las premisas socioculturales de la cultura mexicana. Los sectores II, en esta ocasión, sí aparecían en una posición intermedia, aunque en los casos de Nuevo Laredo y Tijuana no mostraban diferencia estadística con los sectores $\mathrm{III} .{ }^{4}$ Mientras que el promedio de respuestas de los sectores III se ubicaba en el límite superior de la opción "de acuerdo", el de los sectores I se manifestaba en el nivel inferior de la opción "tal vez". Así, la hipótesis de que a mayor clase social mayor la lejanía hacia los valores tradicionales de la cultura mexicana, parecía corroborarse.

4 En el caso del sector II de Nuevo Laredo, tampoco se presentaba una diferencia significativa con su sector $\mathrm{l}$. 
CUADRO 5. Análisis de varianza simple de actitudes favorables a Estados Unidos entre los tres sectores socioeconómicos de Nuevo Laredo, Ciudad Juárez, Tijuana y San Luis Potosí: 1987.

\begin{tabular}{|c|c|c|c|c|}
\hline Estrato & $\begin{array}{l}\text { Nuevo } \\
\text { Laredo }\end{array}$ & $\begin{array}{l}\text { Ciudad } \\
\text { Juárez }\end{array}$ & Tijuana & $\begin{array}{c}\text { San Luis } \\
\text { Potosí }\end{array}$ \\
\hline Sector I & $\begin{array}{c}8.7^{\mathrm{a}} \\
2.2^{\mathrm{b}} \\
(3.4)^{\mathrm{c}} \\
\mathrm{n}=346\end{array}$ & $\begin{array}{c}8.5 \\
2.1 \\
(2.5) \\
n=325\end{array}$ & $\begin{array}{c}8.9 \\
2.2 \\
(2.7) \\
\mathrm{n}=193\end{array}$ & $\begin{array}{c}8.0 \\
2.0 \\
(2.1) \\
\mathrm{n}=306\end{array}$ \\
\hline Sector II & $\begin{array}{c}9.0 \\
2.2 \\
(2.6) \\
\mathrm{n}=598\end{array}$ & $\begin{array}{c}8.3 \\
2.1 \\
(2.2) \\
\mathrm{n}=705\end{array}$ & $\begin{array}{c}9.1 \\
2.2 \\
(2.4) \\
n=530\end{array}$ & $\begin{array}{c}8.7 \\
2.2 \\
(2.8) \\
\mathrm{n}=554\end{array}$ \\
\hline Sector III & $\begin{array}{c}10.1 \\
2.5 \\
(3.0) \\
n=428\end{array}$ & $\begin{array}{c}8.4 \\
2.1 \\
(2.4) \\
n=568\end{array}$ & $\begin{array}{c}9.0 \\
2.2 \\
(2.6) \\
\mathrm{n}=493\end{array}$ & $\begin{array}{c}9.0 \\
2.2 \\
(2.9) \\
\mathrm{n}=676\end{array}$ \\
\hline Valor de F & 25.6 & .45 & $.47^{\circ}$ & 15.8 \\
\hline $\begin{array}{l}\text { Significancia } \\
\text { de la F }\end{array}$ & $.0000^{\mathrm{d}}$ & $.6398^{\mathrm{e}}$ & $.6256^{\mathrm{f}}$ & $.0000^{\mathrm{g}}$ \\
\hline \multicolumn{5}{|c|}{ 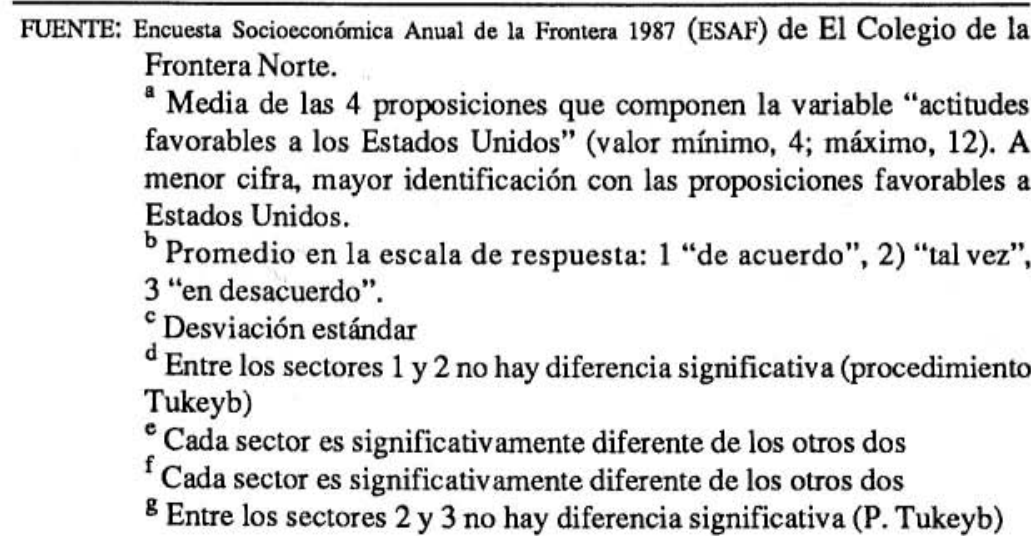 } \\
\hline
\end{tabular}


CUADRO 6. Análisis de varianza simple de actitudes hacia las premisas socioculturales entre los tres sectores socioeconómicos de Nuevo Laredo, Ciudad Juárez, Tijuana y San Luis Potosí: 1987.

\begin{tabular}{lcccc}
\hline Estrato & $\begin{array}{c}\text { Nuevo } \\
\text { Laredo }\end{array}$ & $\begin{array}{c}\text { Ciudad } \\
\text { Juárez }\end{array}$ & Tijuana & $\begin{array}{c}\text { San Luis } \\
\text { Potosí }\end{array}$ \\
\hline Sector I & $50.9^{\mathrm{a}}$ & 59.7 & 56.7 & 54.6 \\
& $2.8^{\mathrm{b}}$ & 3.3 & 3.1 & 3.0 \\
& $(7.4)^{\mathrm{c}}$ & $(12.6)$ & $(8.5)$ & $(7.3)$ \\
& $\mathrm{n}=348$ & $\mathrm{n}=335$ & $\mathrm{n}=193$ & $\mathrm{n}=320$ \\
Sector II & 50.1 & 53.7 & 51.2 & 48.7 \\
& 2.8 & 2.9 & 2.8 & 2.7 \\
& $(8.4)$ & $(8.9)$ & $(8.2)$ & $(8.8)$ \\
Sector III & $\mathrm{n}=604$ & $\mathrm{n}=714$ & $\mathrm{n}=533$ & $\mathrm{n}=571$ \\
& 49.1 & 50.7 & 50.6 & 47.7 \\
& 2.7 & 2.8 & 2.8 & 2.6 \\
& $(7.7)$ & $(7.3)$ & $(12.3)$ & $(7.8)$ \\
Valor de F & $\mathrm{n}=433$ & $\mathrm{n}=569$ & $\mathrm{n}=499$ & $\mathrm{n}=690$ \\
& 5.0 & 97.0 & 26.4 & 83.4 \\
Significancia & & & & \\
de la F & $.0066^{\mathrm{d}}$ & $.0000^{\mathrm{e}}$ & $.0000^{\mathrm{f}}$ & $.0000^{\mathrm{g}}$ \\
& & & &
\end{tabular}

FUENTE: Encuesta Socioeconómica Anual de la Frontera 1987 (ESAF) de El Colegio de la Frontera Norte.

${ }^{a}$ Media de las 18 proposiciones que componen la escala de premisas socioculturales (valor mínimo, 18; máximo, 90). A menor cifra, mayor identificación con las proposiciones de Díaz Guerrero sobre temor a la autoridad.

b Promedio en la escala de respuesta: 1 "muy de acuerdo", 2 "de acuerdo", 3 "tal vez", 4 "en desacuerdo" y 5 "muy en desacuerdo".

"Desviación estándar de la media para las 18 proposiciones

${ }^{\mathrm{d}}$ Sólo hay diferencia significativa entre los sectores 1 y 3 (procedimiento Tukeyb)

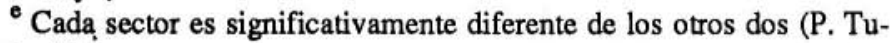
keyb).

${ }^{f}$ Entre los sectores 2 y 3 no hay diferencia significativa (P. Tukeyb).

${ }^{8}$ Cada sector es significativamente diferente de los otros dos (P.Tukeyb) 
CUADRO 7. Prueba t de actitudes favorables al sistema capitalista entre los hombres y mujeres de Nuevo Laredo, Ciudad Juárez, Tijuana y San Luis Potosí: 1987.

\begin{tabular}{lcccc}
\hline Sexo & $\begin{array}{c}\text { Nuevo } \\
\text { Laredo }\end{array}$ & $\begin{array}{c}\text { Ciudad } \\
\text { Juárez }\end{array}$ & Tijuana & $\begin{array}{c}\text { San Luis } \\
\text { Potosí }\end{array}$ \\
\hline Masculino & $19.1^{\mathrm{a}}$ & 16.9 & 17.3 & 17.1 \\
& $2.1^{\mathrm{b}}$ & 1.9 & 1.9 & 1.9 \\
& $(5.3)^{\mathrm{c}}$ & $(4.3)$ & $(4.2)$ & $(4.3)$ \\
& $\mathrm{n}=657$ & $\mathrm{n}=749$ & $\mathrm{n}=601$ & $\mathrm{n}=721$ \\
Femenino & 20.6 & 17.9 & 19.8 & 18.8 \\
& 2.3 & 2.0 & 2.2 & 2.1 \\
& $(5.8)$ & $(4.6)$ & $(5.3)$ & $(5.0)$ \\
Valor de t & $\mathrm{n}=728$ & $\mathrm{n}=869$ & $\mathrm{n}=624$ & $\mathrm{n}=860$ \\
Significancia & -5.01 & -4.54 & -8.83 & -7.46 \\
de la t & .000 & .000 & .000 & .000 \\
\hline
\end{tabular}

FUENTE: Encuesta Socioeconómica Anual de la Frontera 1987 (ESAF) de El Colegio de la Frontera Norte.

${ }^{\text {a }}$ Media de las 9 proposiciones que componen la variable "actitudes procapitalistas" (valor mínimo, 9; máximo, 21). A menor cifra, mayor identificación con las proposiciones favorables al capitalismo.

b Promedio en la escala de respuesta: 1 "de acuerdo", 2 "tal vez", 3 "en desacuerdo".

"Desviación estándar

En cuanto a la variable género, los cuadros 7 y 8 muestran que los hombres, en todas las poblaciones, tendían a manifestar actitudes significativamente más favorables que las mujeres hacia el sistema capitalista y hacia Estados Unidos. En el primer caso, las respuestas de los hombres promediaban en la parte superior de la opción "de acuerdo" mientras que las de las mujeres se ubican en "tal vez". En cuanto a actitudes hacia Estados Unidos, ambos grupos tenían sus promedios en la parte inferior de la opción "tal vez", a pesar de las diferencias significativas entre hombres y mujeres. De este modo, las hipótesis de que los hombres tienden a manifestar actitudes más favorables que las mujeres hacia el modo de producción capitalista y hacia los Estados Unidos resultaban corroboradas. 
CUADRO 8. Prueba t de actitudes favorables a los Estados Unidos entre hombres y mujeres de Nuevo Laredo, Ciudad Juárez, Tijuana y San Luis Potosí: 1987.

\begin{tabular}{|c|c|c|c|c|}
\hline Sexo & $\begin{array}{l}\text { Nuevo } \\
\text { Laredo }\end{array}$ & $\begin{array}{l}\text { Ciudad } \\
\text { Juárez }\end{array}$ & Tijuana & $\begin{array}{c}\text { San Luis } \\
\text { Potosí }\end{array}$ \\
\hline Masculino & $\begin{array}{c}8.9^{\mathrm{a}} \\
2.2^{\mathrm{b}} \\
(2.7)^{\mathrm{c}} \\
\mathrm{n}=648\end{array}$ & $\begin{array}{c}8.0 \\
2.0 \\
(2.0) \\
\mathrm{n}=739\end{array}$ & $\begin{array}{c}8.5 \\
2.1 \\
(2.1) \\
\mathrm{n}=597\end{array}$ & $\begin{array}{c}8.3 \\
2.1 \\
(2.3) \\
\mathrm{n}=705\end{array}$ \\
\hline Femenino & $\begin{array}{c}9.5 \\
2.4 \\
(2.2) \\
n=724\end{array}$ & $\begin{array}{c}8.7 \\
2.2 \\
(2.6) \\
\mathrm{n}=859\end{array}$ & $\begin{array}{c}9.5 \\
2.4 \\
(2.8) \\
n=619\end{array}$ & $\begin{array}{c}9.1 \\
2.3 \\
(3.0) \\
\mathrm{n}=831\end{array}$ \\
\hline Valor de $\mathrm{t}$ & -3.78 & -6.52 & -7.29 & -5.87 \\
\hline Significancia & .000 & .000 & .000 & .000 \\
\hline \multicolumn{5}{|c|}{$\begin{array}{l}\text { FUENTE: Encuesta Socioeconómica Anual de la Frontera } 1987 \text { (ESAF) de El } \\
\text { Colegio de la Frontera Norte. } \\
\text { "Media de las } 4 \text { proposiciones que componen la variable "actitudes } \\
\text { favorables a Estados Unidos" (valor mínimo, 4; máximo, 12). A menor } \\
\text { cifra, mayor identificación con las proposiciones favorables a Estados } \\
\text { Unidos. } \\
\text { 'Promedio en la escala de respuesta: } 1 \text { "de acuerdo", } 2 \text { "tal vez", } \\
3 \text { "en desacuerdo". } \\
\text { "Desviación estándar de la media para las 4 proposiciones. }\end{array}$} \\
\hline
\end{tabular}

En cuanto a la cercanía con las premisas socioculturales, el cuadro 9 resulta sumamente interesante. Mientras que entre los hombres y las mujeres de San Luis Potosí no existía diferencia significativa en su proximidad con las proposiciones tradicionales, en tres ciudades fronterizas las mujeres manifestaban mayor lejanía hacia dichas premisas que los hombres. Sin embargo, los promedios de ambos grupos, en la frontera, se ubican en la parte superior de la opción "de acuerdo" (casos de Tijuana y Nuevo Laredo) o apenas alcanzaban a colocarse en el "tal vez" (caso de Ciudad Juárez), por lo que la distancia entre hombres y mujeres fronterizos, aunque significativa estadísticamente, no refleja diferencias radicales. En contraste, los promedios 
CUADRO 9. Prueba $t$ de las actitudes hacia la escala de premisas socioculturales entre hombres y mujeres de Nuevo Laredo, Ciudad Juárez, Tijuana y San Luis Potosí: 1987.

\begin{tabular}{|c|c|c|c|c|}
\hline Sexo & $\begin{array}{l}\text { Nuevo } \\
\text { Laredo }\end{array}$ & $\begin{array}{l}\text { Ciudad } \\
\text { Juárez }\end{array}$ & Tijuana & $\begin{array}{c}\text { San Luis } \\
\text { Potosí }\end{array}$ \\
\hline Masculino & $\begin{array}{c}49.1^{\mathrm{a}} \\
2.7^{\mathrm{b}} \\
(7.6)^{\mathrm{c}} \\
\mathrm{n}=657\end{array}$ & $\begin{array}{c}53.3 \\
2.9 \\
(10.7) \\
\mathrm{n}=749\end{array}$ & $\begin{array}{c}51.0 \\
2.8 \\
(10.8) \\
\mathrm{n}=601\end{array}$ & $\begin{array}{c}36.8 \\
2.0 \\
(7.1) \\
\mathrm{n}=721\end{array}$ \\
\hline Femenino & $\begin{array}{c}50.8 \\
2.8 \\
(8.2) \\
\mathrm{n}=728\end{array}$ & $\begin{array}{c}54.3 \\
3.0 \\
(9.1) \\
\mathrm{n}=869\end{array}$ & $\begin{array}{c}52.7 \\
2.9 \\
(9.8) \\
\mathrm{n}=624\end{array}$ & $\begin{array}{c}36.9 \\
2.0 \\
(6.2) \\
\mathrm{n}=860\end{array}$ \\
\hline Valor de $\mathrm{t}$ & -4.00 & -1.99 & -2.89 & -0.33 \\
\hline $\begin{array}{l}\text { Significancia } \\
\text { de la } t\end{array}$ & $.000^{\mathrm{d}}$ & $.047^{\mathrm{e}}$ & $.004^{\mathrm{f}}$ & $.744^{\mathrm{g}}$ \\
\hline \multicolumn{5}{|c|}{$\begin{array}{l}\text { FUENTE: Encuesta Socioeconómica Anual de la Frontera } 1987 \text { (ESAF) de El } \\
\text { Colegio de la Frontera Norte. } \\
\text { a Media de las } 18 \text { proposiciones que componen la escala de premisas } \\
\text { socioculturales (valor mínimo, 18; máximo, 90). A menor cifra, } \\
\text { mayor identificación con las proposiciones favorables a Estados } \\
\text { Unidos } \\
\text { b Promedio en la escala de respuesta: } 1 \text { "muy de acuerdo", } 2 \text { "de } \\
\text { acuerdo", } 3 \text { "tal vez", } 4 \text { "en desacuerdo" y } 5 \text { "muy en desacuerdo". } \\
\text { c Desviación estándar } \\
\text { d Sólo hay diferencia significativa entre los sectores } 1 \text { y } 3 \text { (procedi- } \\
\text { miento Tukeyb) } \\
\text { ¿ Cada sector es significativamente diferente de los otros dos (P. } \\
\text { Tukeyb). } \\
\text { f Entre los sectores } 2 \text { y } 3 \text { no hay diferencia significativa (P. Tukeyb). } \\
\text { g Cada sector es significativamente diferente de los otros dos (P. } \\
\text { Tukeyb) }\end{array}$} \\
\hline
\end{tabular}


de respuesta de los hombres y mujeres de San Luis Potosí se ubicaban en el límite del "muy de acuerdo" con el "de acuerdo" (2.0), lo que indicaba un mayor grado de cercanía por género en esa población del interior del país a las premisas socioculturales. De esta forma, la hipótesis de que las mujeres tienden a identificarse más que los hombres con dichas proposiciones quedó rechazada para el caso de las poblaciones fronterizas. De hecho, el género resultó un predictor útil para cl caso contrario, es decir, los hombres tienden a identificarse más con las premisas de Díaz Guerrero que las mujeres. Para el caso de San Luis Potosí, sin embargo, el sexo no demostró constituir un predictor válido, ya que no se encontraron diferencias significativas entre los hombres y las mujeres de dicha población.

\section{CONCLUSIONES}

Mediante el análisis de las escalas de actitudes sobre el sistema capitalista, sobre los Estados Unidos y sobre las premisas socioculturales definidas por Rogelio Díaz Guerrero como características de elementos de la cultura tradicional mexicana, el presente trabajo ha pretendido determinar si la proximidad geográfica con los Estados Unidos propicia diferencias significativas entre los habitantes de la frontera norte y los del interior del país.

Los resultados discutidos arriba tienden a rebatir la creencia de que en lafrontera existe mayor identificación con Estados Unidos y con su sistema económico capitalista que en el interior del país. Es significativo, por ejemplo, que los habitantes de Tijuana y Nuevo Laredo manifestaran una percepción menos favorable del sistema capitalista y de Estados Unidos que la de los residentes de San Luis Potosí. Así, a pesar de estar más lejanos con Estados Unidos, la muestra de potosinos encuestada tendía a percibir con mayor simpatía al vecino del norte y a su sistema económico que quienes convivian diariamente con instituciones y personas de ese país en la franja divisoria. Y aunque los habitantes de Ciudad Juárez parecían mantener las actitudes más favorables de las cuatro ciudades hacia Estados Unidos y hacia el modo de producción capitalista, sus respuestas se ubicaban en el punto medio de la escala, es decir, en el "tal vez", y no en la aprobación incondicional.

Aunque los hallazgos para la escala de premisas socioculturales no son tan contundentes como para rechazar que existan diferencias entre la frontera norte y el interior del país, sí demuestran que la proximidad geográfica con los Estados Unidos no representaba una pérdida de los valores tradicionales definidos por la escala de Dfaz Guerrero. A nivel general, seadvierte que por lo menos una ciudad fronteriza, Nuevo Laredo, no presentaba diferencias significativas con San Luis Potosí en la cercanía 
a las creencias tradicionales sobre las relaciones familiares y el machismo. Tijuana y Ciudad Juárez, estadísticamente menos cercanas a dichas premisas, seguían ubicando el promedio de sus respuestas en la categoría "de acuerdo", lo que indica que sus diferencias no llegaban al grado de poner las afirmaciones en duda o contradecirlas.

De esta manera, los hallazgos reflejan de cierto modo la complejidad y las limitaciones de los conceptos que pretenden definir la identidad cultural de los mexicanos. Si por ésta se entiende la permanencia de valores familiares tradicionales como la obediencia y el respeto a los padres, o de elementos psicosociales como el machismo, entonces los fronterizos con excepción de los neolaredenses- sí parecerían reflejar una menor identificación, aunque no al grado de rechazar las premisas tradicionales (las respuestas de los tijuanenses y juarenses se ubicaban en el nivel "de acuerdo"). Si por erosión de la identidad cultural se entiende manifestar actitudes favorables hacia los Estados Unidos y hacia el modo de producción capitalista -en el sentido de afirmaciones como "Las elecciones de México deberían ser como las de Estados Unidos" o "los mejores gobernantes serían los empresarios" - resulta que los habitantes de San Luis Potosí reflejan una mayor pérdida de "identidad" que los mismos habitantes de Tijuana y Nuevo Laredo.

Las diferencias entre las tres poblaciones fronterizas, por otra parte, seffalan la necesidad de profundizar en los análisis particulares de cada población y de reconocer que existen dinámicas culturales significativamente diferentes entre ellas, lo que impide realizar generalizaciones sobre lo que ocurre en la frontera norte en esta área. Uno de los retos que deberá enfrentar la investigación sobre identidad cultural en esta región del país será analizar las causas que provocan que la población de Nuevo Laredo presente grados de cercanía con las premisas socioculturales y actitudes menos favorables al sistema capitalista y a Estados Unidos que Tijuana y Ciudad Juárez —e incluso que San Luis Potosí-, y que Ciudad Juárez, consistentemente, manifieste la mayor identificación con Estados Unidos y su sistema económico y la mayor lejanía con las creencias tradicionales definidas por Díaz Guerrero.

Para el debate sobre el posible impacto en la identidad nacional del acuerdo de libre comercio entre México y el vecino del norte, sin embargo, parece claro que la experiencia fronteriza y la mayor vinculación economica de esa zona con Estados Unidos no ha erosionado en sus habitantes actitudes y valores tan importantes como los analizados aquí. Es muy significativo que los fronterizos, pese a su histórica vinculación económica con los Estados Unidos y a su permanente contacto con las instituciones, valores y actitudes de ese país, manifiesten percepciones menos favorables 
hacia él que los habitantes de una ciudad del interior de México como San Luis Potosf, con grados mucho menores de contacto con la nación vecina. En este sentido, resultan válidas las observaciones de Bustamante sobre el refuerzo de la identidad nacional en los fronterizos debido a su interacción con "los otros" en un contexto donde se facilita diferenciar a las dos culturas. ${ }^{5}$

En cuanto a la importancia de la variable clase social en la predicción de una mayor o menor cercanía a premisas tradicionales de la cultura mexicana y a una mayor o menor identificación con los Estados Unidos y su sistema económico, resulta muy clara su aplicación. En todos los casos, las diferencias entre los sectores I y III de cada ciudad fueron estadísticamente significativas. Así, la variable clase social constituía un predictor mucho más útil y válido para detectar diferencias en el grado de identidad nacional de los mexicanos que la proximidad geográfica con los Estados Unidos. El señalamiento de diversos teóricos sociales sobre la identificación de las clases hegemónicas con el sistema capitalista y con Estados Unidos, así como la persistencia de elementos tradicionales en las clases bajas debido a su situación de subordinación, parecía encontrar apoyo en los hallazgos reportados aquí. El comportamiento irregular de los sectores II-en ocasiones identificándose más con las actitudes del sector I, en otras con las del sector III- muestra la compleja dinámica cultural que existe en ellas y el proceso de transición por la que atraviesan. Investigaciones posteriores deberán prestar particular atención a esta complejidad para determinar más claramente qué rol juega la pertenencia a este nivel sociocconómico en la conformación de actitudes culturales.

Algo similar puede señalarse con respecto al sexo. ¿A qué se debe que las mujeres fronterizas manifiesten actitudes menos favorables hacia los Estados Unidos y su modo de producción capitalista y que, al mismo tiempo, presenten mayor lejanía hacia premisas socioculturales como las incluidas en este estudio? ¿Qué fenómenos psicológicos, sociales y probablemente económicos propician esta bifurcación en sus actitudes culturales? Las preguntas anteriores incrementan su importancia al advertir que entre los hombres y las-mujeres de San Luis Potosín no se manifestaban diferencias significativas, sólo entre los grupos fronterizos. Por lo pronto, el presente trabajo sugiere la necesidad de incluir al género como variable independiente en futuras investigaciones.

5 Dice Bustamante: “(...) la otredad de lo estadounidense ayuda en la frontera norte a definir lo mexicano. Parad6jicamente, la vecindad con el extranjero le da al fronterizo una ventaja en su identidad étnica, frente a los mexicanos del interior del país donde no es tan inmediata y cotidiana esa experiencia de otredad" (Bustamante, 1989:23). 


\section{BIBLIOGRAFÍA}

BONFIL Batalla, Guillermo. 1982. "De culturas populares y política cultural", en: Culturas populares, politica cultural. México. Museo de Culturas Populares/sEP.

BUSTAMANTE, Jorge A. 1983. Tensiones sociales en la frontera norte y en la Ciudad de México. Tijuana, mimeo.

BUSTAMANTE, Jorge. 1989. "Frontera México-Estados Unidos: reflexiones para un marco teórico", en: Frontera Norte. Vol. 1, no. 1, El Colegio de la Frontera Norte.

CASTAÑEDA, Jorge G. 1989. "El infundado temor a la norteamericanización", en Jorge G. Castaf́eda y Robert A. Pastor. LImites en la amistad: México y Estados Unidos. México: Joaquín Mortiz/Planeta.

DESAULNIERS, Jean-Pierre. 1987. "What does Canada Want? or L'historie san lecon". Media, Culture and Society. Vol. 9.

DURAN, Leonel. 1984. "Cultura popular y mentalidades populares", en: Adolfo Colombres (ed.) La cultura popular, México, Premiá Editores.

GARCÍA Canclini, Néstor. 1987. "¿De qué estamos hablando cuando hablamos de lo popular?", en: Comunicación y culturas populares en Latinoamérica (Seminario del Consejo Latinoamericano de Ciencias Sociales). México FELAFAcs/Gustavo Gili.

GONZÁLEZ Ramírez, Raúl y Jorge Santibáñez Romellón. 1990. "La encuesta socioeconómica anual de la frontera (ESAF), nota descriptiva". Estudios sociológicos VIII, no. 23.

STAVENHAGEN, Rodolfo. 1984. "La cultura popular y la creación intelectual", en: Adolfo Colombres (ed.). La cultura popular. México, Premiá Editores. 